\title{
Incipient $\mathrm{FeO}(111)$ monolayer formation during 0 - adsorption on $\mathrm{Fe}(110)$ surface
}

DOI:

10.1016/j.commatsci.2017.03.033

\section{Document Version}

Accepted author manuscript

Link to publication record in Manchester Research Explorer

\section{Citation for published version (APA):}

Chohan, U., Koehler, S., \& Jimenez-Melero, E. (2017). Incipient FeO(111) monolayer formation during Oadsorption on Fe(110) surface. Computational Materials Science, 134, 109-115.

https://doi.org/10.1016/j.commatsci.2017.03.033

\section{Published in:}

Computational Materials Science

\section{Citing this paper}

Please note that where the full-text provided on Manchester Research Explorer is the Author Accepted Manuscript or Proof version this may differ from the final Published version. If citing, it is advised that you check and use the publisher's definitive version.

\section{General rights}

Copyright and moral rights for the publications made accessible in the Research Explorer are retained by the authors and/or other copyright owners and it is a condition of accessing publications that users recognise and abide by the legal requirements associated with these rights.

\section{Takedown policy}

If you believe that this document breaches copyright please refer to the University of Manchester's Takedown Procedures [http://man.ac.uk/04Y6Bo] or contact uml.scholarlycommunications@manchester.ac.uk providing relevant details, so we can investigate your claim.

\section{OPEN ACCESS}




\title{
Incipient $\mathrm{FeO}(111)$ monolayer formation during O-adsorption on
}

\section{Fe(110) surface}

Urslaan K. Chohan ${ }^{\mathrm{a}, \mathrm{c}, *}$, Sven P. K. Koehler ${ }^{\mathrm{b}, \mathrm{c},}$, Enrique Jimenez-Melero $^{\mathrm{a}, \mathrm{c}}$

\author{
${ }^{a}$ School of Materials, The University of Manchester, Manchester M13 9PL, UK \\ ${ }^{\mathrm{b}}$ School of Chemistry, The University of Manchester, Manchester M13 9PL, UK \\ ${ }^{c}$ Dalton Cumbrian Facility, The University of Manchester, Moor Row CA24 3HA, UK \\ ${ }^{\mathrm{d}}$ Photon Science Institute, The University of Manchester, Manchester M13 9PL, UK
}

*Corresponding author.

Email: urslaan.chohan@postgrad.manchester.ac.uk

University of Manchester

School of Materials

Oxford Road

Manchester

M13 9PL

United Kingdom 


\begin{abstract}
The adsorption of $\mathrm{O}$ atoms on the $\mathrm{Fe}(110)$ surface has been investigated by density functional theory for increasing degrees of oxygen coverage from 0.25 to 1 monolayer, to follow the evolution of the $\mathrm{O}-\mathrm{Fe}(110)$ system into an $\mathrm{FeO}(111)$-like monolayer. We found that the quasi-threefold site is the most stable adsorption site for all coverages, with adsorption energies of $\sim 2.8$ to $4.0 \mathrm{eV}$ per $\mathrm{O}$ atom. Oxygen adsorption results in surface geometrical changes such as interlayer relaxation and buckling, the latter of which decreases with coverage. The calculated vibrational frequencies range from $265-470 \mathrm{~cm}^{-1}$ for the frustrated translational modes and $480-620 \mathrm{~cm}^{-1}$ for the stretching mode, and hence are in good agreement with the experimental values reported for bulk $\mathrm{FeO}$ wüstite. The hybridization of the oxygen $2 p$ and iron $3 d$ orbitals increases with oxygen coverage, and the partial density of states for the $\mathrm{O}-\mathrm{Fe}(110)$ system at full coverage resembles the one reported in the literature for bulk FeO. These results at full oxygen coverage point to the incipient formation of an $\mathrm{FeO}(111)$-like monolayer that would eventually lead to the bulk FeO oxide layer.
\end{abstract}

Keywords: Density functional theory, ferrite, oxidation, chemisorption, partial density of states, surface relaxation 


\section{Introduction}

The study of oxide formation on metallic surfaces is of great relevance in a wide range of technological applications, such as heterogeneous catalysis [1], oxide electronics [2] or nuclear reactor technologies [3]. In all these cases, the formation of an oxide on metallic surfaces is a sequential process. Initially, the surface exposure to oxygen results in the dissociative chemisorption of the oxygen molecules, resulting in a monolayer formation of oxygen atoms on the surface [4-6]. The metal surface atoms act as electron donors to these oxygen atoms [7]. With continuous exposure, this process eventually leads to the nucleation and growth of relatively thick surface oxide films. The incorporation and gradual diffusion of additional oxygen ions into the bulk structure, in parallel with the diffusion of metal cations from the bulk into the newly-formed oxide layer $[5,6,8,9]$, lead progressively to a compact bulk oxide [10]. It is important to note that an understanding of the initial O-chemisorption process and its transition into the surface oxide provides a first insight into the process of oxide formation in metallic systems.

In this study, we focussed on ferritic steels, which are present in various technological components where oxidation phenomena can compromise their structural integrity and limit their service life, such as in current power plants and potentially in advanced nuclear reactors concepts [11]. The low-index (110) plane of $\alpha$-Fe is of particular interest, due to it being the most densely packed plane. The oxidation of this plane has been the subject of various studies through a plethora of methods, both theoretical and experimental, yet the initial stages of $\mathrm{FeO}$ formation are not yet fully understood. $\mathrm{FeO}$ wüstite is stable at temperatures above $570^{\circ} \mathrm{C}$ and can coexist with magnetite $\mathrm{Fe}_{3} \mathrm{O}_{4}$ at high oxygen levels $[12,13]$. Linear kinetics has been reported for the oxidation of iron at low oxygen potentials where only $\mathrm{FeO}$ forms. The mobility of cations and electrons in $\mathrm{FeO}$ is relatively high [13]. As the wüstite scale thickens, 
the diffusion rate through it decreases until a critical thickness is reached when diffusion becomes rate-controlling and the kinetics parabolic [12]. In this work we address the incipient formation of $\mathrm{FeO}$ from the initial oxygen chemisorption at the $\mathrm{Fe}(110)$ surface as the O-coverage increases, and before the $\mathrm{FeO}$ layer starts to grow and thicken.

There is significant scatter in the literature regarding the O-adsorption site and layer geometry for increasing coverage, and consequently regarding the vibrational frequencies for oxygen-covered iron, with a wide range of values measured, but not always with a clear assignment of the type of vibrational mode. A range of sticking coefficients for the adsorption of $\mathrm{O}_{2}$ on $\mathrm{Fe}(110)$ were measured in various experiments, but later experiments were in agreement of a sticking coefficient of $\sim 1$ for low exposures, which decreased to $\sim 0.05$ for exposures close to 1 Langmuir (L) exposure [4, 14-16]. The earliest low energy electron diffraction (LEED) study investigated the mechanism of oxide layer formation on a single crystal of $\mathrm{Fe}(110)$ [17]. The $(2 \times 2)$ and $(3 \times 1)$ motifs were observed to form sequentially at 0.2 to 1.7 and 4 to $6 \mathrm{~L}$, respectively. The authors also noted the formation of a $\mathrm{FeO}(111)$-type structure at $18.5 \mathrm{~L}$, which transitioned into a reconstructed quasi-hexagonal $(5 \times 12)$ phase following an annealing step at $620 \mathrm{~K}$. This sequence of formation was also observed by other groups $[15,18-20]$, although Weissenrieder et al. indicated the formation of an additional $(2 \times 5)$ structure at low coverages [21]. In various studies the $\mathrm{FeO}(111)$ oxide layer was seen to form parallel to the $\mathrm{Fe}(110)$ surface, with a 4-6\% in-plane expansion in this oxide layer relative to bulk $\mathrm{FeO}[7,22,23]$. Sakisaka et al. studied the band structure of the $(2 \times 2)$ and (3×1) structures using angle-resolved photoemission spectroscopy [24]. They found that the $(2 \times 2)$ structure had negligible dispersion, however, the $(3 \times 1)-\mathrm{O}$ overlay exhibited a strong dispersion of $\sim 1.6 \mathrm{eV}$, caused by O-Fe-O interactions. Spin- and angle-resolved photoemission and LEED experiments by Kim et al. agreed with that assignment [25]. A variety of studies employing a range of techniques were used to measure the Fe-O stretches 
in $\mathrm{FeO}$. An early investigation was conducted by Poling using transmission infrared spectroscopy on oxide powder samples. Two stretches were observed for $\mathrm{FeO}$ at $\sim 425 \mathrm{~cm}^{-1}$ and $\sim 580 \mathrm{~cm}^{-1}$ [26]. Erley and Ibach used high-resolution electron energy loss spectroscopy (HREELS) study to measure the vibrational frequencies of oxygen overlays for varying oxygen exposures [27]. They deduced that $\mathrm{O}$ atoms adsorb at long-bridge (lb) sites for low exposure, with a single vibration at around $500 \mathrm{~cm}^{-1}$. The low frequency relative to a free $\mathrm{O}_{2}$ molecule indicates that the $\mathrm{O}_{2}$ undergoes dissociative chemisorption on the surface. After increasing the exposure, they observed an additional vibration at $200 \mathrm{~cm}^{-1}$, correlating with a $(2 \times 2)$ oxygen overlay $(0.50 \mathrm{ML})$. At still higher exposures, a $(3 \times 1)$ structure $(0.67 \mathrm{ML})$ formed with $\mathrm{O}$ at $\mathrm{lb}$ sites, yielding vibrational stretches at $500-550 \mathrm{~cm}^{-1}$. At the highest exposures and following annealing at $750 \mathrm{~K}$, the above mentioned $(5 \times 12)$ overlay formed, with stretches at $400 \mathrm{~cm}^{-1}$ and $500-550 \mathrm{~cm}^{-1}$, where the $\mathrm{O}$ atoms adsorbed at the quasithreefold (3f) sites. Faria et al. used Raman spectroscopy to measure the Fe-O phonon interactions in pressed pellets of wüstite, and observed a broad peak at $\sim 652 \mathrm{~cm}^{-1}$ [28].

Theoretical calculations using density functional theory (DFT) of the adsorption of $\mathrm{O}$ atoms on the $\mathrm{Fe}(110)$ surface were first reported in the early 2000s. These DFT studies primarily investigated the $(2 \times 2),(3 \times 1)$ and $(1 \times 1)$ geometries [29-35]. There is consensus in the literature regarding the adsorption energy, which linearly increased from $\sim 1.40 \mathrm{eV}$ to $3.50 \mathrm{eV}$ per $\mathrm{O}$ atom from low to high coverage [30, 34]. However, there is disagreement regarding the stability of the four high symmetry adsorption sites (see Fig. 1). In the earlier studies, it was deduced that the most energetically stable adsorption site is the long-bridge (lb) site, while the on-top (ot), short-bridge (sb) and quasi-threefold (3f) sites were found to be unstable [29-31]. This was in contrast to a study by Błoński et al. in which the $3 f$ site was found to be the most stable [32]. However, they attributed this to using a $(3 \times 3)$ geometry, unlike in the prior study which employed a $(2 \times 2)$ geometry. In a calculation by Tan, Zhou 
and Peng, the authors assumed that the oxygen adsorbate resides at the lb site [33]. From their vibrational analysis, only the $(2 \times 2)$ and $(3 \times 1)$ structures were found to be stable. Most notably, they found the $(1 \times 1)$ structure to be a transition state. They did not comment upon this discrepancy beyond stating it is "not stable", though this instability indicates that the oxygen atoms do not reside at the $\mathrm{lb}$ site at the highest coverage. Ossowski and Kiejna, however, indicated that for all coverages, the $3 \mathrm{f}$ site is most stable, with a small energy difference at low coverages between the $\mathrm{lb}$ and $3 \mathrm{f}$ sites [34]. Most recently, Freindl et al. conducted a combined DFT and experimental study [35]. They discovered a new (3×2) phase using LEED, corresponding to a 0.67 ML coverage, and verified its existence using DFT. The 3f site was found to be most stable for this structure.

In this work, we report an in-depth analysis into the adsorption of oxygen on the bcc $\mathrm{Fe}(110)$ surface with increasing coverages using density functional theory. The combined interpretation of the absorption energies and vibrational frequencies allows to unequivocally determine the most stable adsorption site for representative degrees of coverage, and also to interpret the reported experimental values of vibrational frequencies. Changes in the spinpolarized partial density of states (PDOS) of oxygen adsorption on $\mathrm{Fe}(110)$, together with surface geometric effects due to adsorption such as interlayer spacing and buckling, are also discussed. This study considers a range of increasing O-coverage from 0.25 to $1 \mathrm{ML}$, hence we effectively probe the onset of oxidation up to the point where the calculated vibrational spectra and the partial density of states resemble those corresponding to $\mathrm{FeO}(111)$ films.

\section{Computational methodology}

Ab initio calculations were performed based on density-functional theory (DFT), using the Cambridge Serial Total Energy Package (CASTEP) electronic structure code [36]. The Perdew-Burke-Ernzerhof (PBE) framework described the exchange and correlation effects [37], and spin polarization was applied to accurately simulate the ferromagnetic 
properties of $\alpha$-Fe. CASTEP generated on-the-fly ultrasoft pseudopotentials for the Fe and O atoms. A slab model was used to represent a $\mathrm{Fe}(110)$ surface using six representative cell geometries; $(2 \times 2),(3 \times 1),(2 \times 1),(2 \times 2)-2 \mathrm{O},(3 \times 1)-2 \mathrm{O}$, and $(1 \times 1)$. Six layers and a vacuum gap of $20 \AA$ were applied (see previous DFT study for further details [38]). The Monkhorst-Pack (MP) algorithm was employed for $k$ point sampling [39]. A cut-off energy of $750 \mathrm{eV}$ and $16 \times 8 \times 1$ MP grid size yielded converged energies for the $(2 \times 1)$ geometry. The MP grid size was rescaled to apply consistent $k$ point sampling for all geometries. The Gaussian smearing scheme was introduced to treat the partial occupancies about the Fermi level, with a smearing width of $0.1 \mathrm{eV}$ [40]. Oxygen atoms were adsorbed to one side of the slab, with dipole effects considered negligible. Only the top three layers and the $\mathrm{O}$ atoms were relaxed, whilst the remaining three layers had their movement constrained. The Broyden-Fletcher-GoldfarbShanno (BFGS) algorithm [41] optimized the geometry, until the force per atom reached less than $0.05 \mathrm{eV} / \AA$.

Two important surface parameters were calculated for the each employed slab geometry and O-adsorption site; the interlayer relaxation, $\Delta_{i j}$ and the adsorption energy, $E_{\text {ads }}$. The interlayer relaxation is given by

$$
\Delta_{i j}=\left(\frac{d_{i j}-d_{0}}{d_{0}}\right) \times 100 \%
$$

where $d_{0}$ is the bulk interlayer spacing and $d_{i j}$ denotes the interlayer spacing between layers $i$ and $j$ in the slab. Values of $\Delta_{i j}$ were obtained for both the O-free and O-adsorbed $\mathrm{Fe}(110)$ surface. The adsorption energies were computed using the expression

$$
F_{\text {ad }}=\frac{1}{N}\left[F_{\text {Ol slab }}-\left(F_{\text {slab }}+\frac{N}{2} F_{\mathrm{O}_{2}}\right)\right]
$$

where $E_{\mathrm{O}+\text { slab }}$ is the total energy of the O-covered slab, $E_{\text {slab }}$ is the total energy of the clean slab, $E_{\mathrm{O}_{2}}$ is the energy of a free $\mathrm{O}_{2}$ molecule (in a $10 \times 10 \times 10 \AA^{3}$ box), and $N$ is the number of 
$\mathrm{O}$ adsorbates per unit cell. The relaxed geometries were carried forward into the frozen phonon calculations, to compute vibrational frequencies of the surface adsorbed $\mathrm{O}$ on high symmetry sites. The Fe slab atoms had constrained motion, whilst the $\mathrm{O}$ atoms were free to move. A finite displacement of $0.01 \AA$ on $\mathrm{O}$ atoms was applied to compute the dynamical matrix, which was then implemented in calculating the vibrational frequencies.

The potential energy surface (PES) was calculated for the adsorption of oxygen on the $\mathrm{Fe}(110)$ plane. A grid was produced of 69 coordinate positions, which mapped the coordinates of $\mathrm{O}$ atoms along the surface. Three Fe substrate layers were allowed to relax, whilst fixing a single Fe atom on the top surface layer to limit surface reconstruction, and concurrently ensuring the energetics in the adsorption process were accurately captured. The $\mathrm{O}$ atom was allowed to relax normal to the plane only. The adsorption energy was calculated at each grid point, using Eq. 2. Energies were calculated relative to the energy at the ot site.

The spin-polarized partial density of states (PDOS), $n^{ \pm}$, was calculated using the OPTADOS code [42]. Adaptive smearing was applied to smooth the PDOS, with a width of $0.4 \mathrm{eV}$ [43]. From the PDOS, the spin-dependent $d$-band centre, $c_{d}^{ \pm}$, was calculated via the relation [44]

$$
c_{\frac{ \pm}{2}}^{ \pm}=\frac{\int_{-\infty}^{\infty} E n^{ \pm}(E) d E}{\int_{-\infty}^{\infty} n^{+}(E) d E}
$$

which was averaged to obtain the $d$-band centre, $c_{d}$, i.e. $c_{d}=0.5\left(c_{d}{ }^{+}+c_{d}{ }^{-}\right)$.

\section{Results and Discussion}

\subsection{Stability of O-adsorption sites}

We have calculated the adsorption energies of oxygen at the four potential sites on the $\mathrm{Fe}(110)$ surface for representative coverages using Eq. 2, see Figure 2a. For all cell geometries, the lowest energy state is the $3 f$ site, though for the $(2 \times 2)$ geometry $(0.25 \mathrm{ML}$ coverage), the $\mathrm{lb}$ and $3 \mathrm{f}$ sites are close in energy. The $\mathrm{sb}$ and especially the ot sites are 
energetically less favorable. These values suggest that the $\mathrm{O}$ atoms would preferentially adsorb on the $3 \mathrm{f}$ sites. As coverage increases, the adsorption energies decrease linearly. This reduction is likely to stem from an increasing repulsion between co-adsorbed $\mathrm{O}$ atoms with increasing coverage. The relative stability between adsorption sites is graphically presented on the potential energy surface plot for the $(2 \times 2)$ geometry, see Fig. 1 . The ot and sb sites stand out as being energetically less favorable, but more importantly, it can be clearly seen that the $\mathrm{lb}$ and the $3 \mathrm{f}$ sites are not only very close in energy, but also very close spatially $(\sim 0.1 \AA)$, with no discernible barrier between them.

After complete relaxation of the slab, the vibrational frequencies of the $\mathrm{Fe}-\mathrm{O}$ bonds were computed using the frozen phonon method, see Table 1. An $\mathrm{O}$ atom was placed at all four possible adsorption sites for three representative cell geometries corresponding to 0.25 , 0.5 and $1 \mathrm{ML}$, in order to observe the stability with respect to coverage. It can be seen that for all coverages, the $\mathrm{sb}$ and $\mathrm{lb}$ sites have a single imaginary frequency and are thus transition states. For 0.25 and $0.5 \mathrm{ML}$, the ot site is a rank two saddle point. For $1 \mathrm{ML}$ coverage, the ot site is a local minimum, which accounts for the real frequencies at this coverage. However, the $3 \mathrm{f}$ site is a true minimum for all coverages, in agreement with our calculated adsorption energies. Only the $3 \mathrm{f}$ site as the most stable site will be considered in the following calculations.

\subsection{Transition to $\mathrm{FeO}(111)$-like monolayer}

Vibrational frequencies were calculated for $0.25,0.33,0.5,0.67$ and $1 \mathrm{ML}$ coverage for different cell geometries, see Table 2. For all coverages, at least three vibrations were observed, classified into two frustrated translational and one stretching mode. The frustrated translational frequencies, $\tilde{v}_{\mathrm{t} 1}$ and $\tilde{v}_{\mathrm{t} 2}$, oscillate parallel to the $\operatorname{Fe}(110)$ plane, whilst the stretching frequency, $\tilde{\nu}_{\mathrm{s}}$ is normal to the plane, i.e. in the [110] direction. The frustrated translational mode $\tilde{v}_{\mathrm{t} 1}$ spans a frequency range from 265 to $465 \mathrm{~cm}^{-1}$, oscillating in the [110] 
direction, and the $\tilde{v}_{\mathrm{t} 2}$ mode from 375 to $470 \mathrm{~cm}^{-1}$, oscillating in the [001] direction. Furthermore, the stretching frequency occurs at higher wavenumbers, i.e. between $480 \mathrm{~cm}^{-1}$ and $620 \mathrm{~cm}^{-1}$. The relative increase in the frequencies for each mode correlates with the increasing coverage.

Fig. $2 \mathrm{~b}$ shows the frequency values determined in our DFT work, and also the experimental values available in the literature for both oxygen adsorbed on $\mathrm{Fe}(110)$ surface and bulk FeO. Our calculated values for full coverage fit the early experiments by Poling et al. rather well [26]. They employed transmission IR spectroscopy of FeO powders, and hence observed what we believe are $\mathrm{Fe}-\mathrm{O}$ stretches along the surface normal at $\sim 580 \mathrm{~cm}^{-1}$ (as compared to our value of $620 \mathrm{~cm}^{-1}$ at full coverage), and also frustrated translational modes parallel to the surface at $\sim 425 \mathrm{~cm}^{-1}$ (as compared to our $464 \mathrm{~cm}^{-1}$ and $466 \mathrm{~cm}^{-1}$; for reasons as to why one or two frequencies are detected, see discussion below) as these transmission IR studies were not restricted by the surface selection rules that limit reflection-absorption infrared spectroscopy studies on metal surfaces. The Raman studies by Faria et al. also detected a vibrational stretch at frequencies comparable to our results $\left(\sim 580 \mathrm{~cm}^{-1}\right.$ as compared to $\left.620 \mathrm{~cm}^{-1}\right)$ [28]. In the HREELS work by Erley and Ibach [27], two oscillations for low $\mathrm{O}_{2}$ exposure (corresponding to the $(2 \times 2)$ geometry) at $200 \mathrm{~cm}^{-1}$ and $500 \mathrm{~cm}^{-1}$ were observed. As coverage increased, the $200 \mathrm{~cm}^{-1}$ stretch was no longer present, leaving a single $500 \mathrm{~cm}^{-1}$ stretch. Erley and Ibach argued that due to the EELS selection rule, namely that only stretching modes perpendicular to the surface are EELS active, the single stretch was necessary to be of at least $\mathrm{C}_{2 \mathrm{v}}$ symmetry [27]. From this it was concluded that the oxygen atom adsorbed at either the on-top or two-fold long-bridge site. However the stretching frequency is far away from that of a free $\mathrm{FeO}$ molecule (at $\sim 880 \mathrm{~cm}^{-1}$ ) [45], and thus preference was given the $2 \mathrm{f}$ site for the adsorbed oxygen. Our analysis indicates that it is 
more likely that the $\mathrm{O}$ atoms occupy the $3 \mathrm{f}$ site. As has been mentioned earlier and as shown in Fig. 1, the $\mathrm{lb}$ site and the $3 \mathrm{f}$ site are only separated by $\sim 0.1 \AA$ spatially, with the $3 \mathrm{f}$ site being surrounded by two potential $\mathrm{lb}$ sites, and both sites are very close in energy $(\Delta E<0.05 \mathrm{eV}$ at $0.25 \mathrm{ML}$ coverage) as per our adsorption energy calculations, hence there is a probability that the $\mathrm{O}$ atom might migrate between the $\mathrm{lb}$ and $3 \mathrm{f}$ sites. At increasing coverages, Erley and Ibach detected vibrations around $500-550 \mathrm{~cm}^{-1}$, which match our stretching frequencies well. This is an encouraging agreement as these studies were performed under specular HREELS conditions which are only sensitive to vibrations along the surface normal, which are stretching frequencies (but not the frustrated translation modes). An additional stretch at $\sim 400 \mathrm{~cm}^{-1}$ at the highest coverage and after annealing of the surface was believed to be due to the formation of $\mathrm{FeO}(111)$ after transitioning from a $(5 \times 12)$ geometry. This bilayer structure was found to have $\mathrm{O}$ atoms at the $3 \mathrm{f}$ sites with an increased $\mathrm{Fe}-\mathrm{O}$ bond length, which seems to be responsible for the reduced vibrational frequency found in their study. Our results for O-coverage between 0.33 and $0.67 \mathrm{ML}$ lie within the band of experimental values for oxygen adsorbed on the $\mathrm{Fe}(110)$ surface, while for full coverage our DFT values are in good agreement with the frequencies determined experimentally for bulk $\mathrm{FeO}$, and therefore point to the formation of an $\mathrm{FeO}(111)$-like monolayer at 1.0 ML.

The similarity between the calculated and experimentally observed vibrations for increasing O-coverage can be explained based on the calculated spin-polarized partial density of states (PDOS) of oxygen adsorption on Fe(110), see Figure 3a-e. There is a shift in the density of states for the oxygen atoms, which is most noticeable in between -7 and $-4 \mathrm{eV}$. Most notably, at the highest coverage, the form of the PDOS for the $\mathrm{O}$ atom begins to resemble the PDOS of the first layer of the Fe. This indicates there is hybridization between the $2 p$ orbital of the $\mathrm{O}$ atom and the $3 d$ orbital of the surface Fe atom. This is further 
elaborated by calculating the $d$-band center, $c_{d}$, as a function of the adsorption energy, $E_{\text {ads }}$, see Fig. 3f. The linear relationship of the two parameters obeys the Hammer-Nørskov $d$-band model [46], which suggests that there is increasing hybridization with increasing coverage, and therefore the $\mathrm{O}$ atoms are becoming more strongly chemisorbed to the surface $\mathrm{Fe}$ atoms. This indicates that the surface layer is beginning to behave more like an oxide overlay at higher coverages. Furthermore, the form of the PDOS resembles those calculated in previous literature for $\mathrm{FeO}$, in particular our calculated PDOS for low coverage is similar to the PDOS produced by Terakura et al. [47]. At high coverage there is resemblance to the PDOS produced by Ye et al. [48] and Rödl et al. [49], i.e. there is a peak alignment and similar form in the -4 to $-7 \mathrm{eV}$ range. Hence there are similarities in the electronic properties between $\mathrm{FeO}$ and our O-Fe(110) structures, especially at higher O-coverage.

\subsection{Surface geometric effects from oxygen adsorption}

The rearrangement of $\mathrm{Fe}$ atoms in the surface layers relative to the equilibrium bulk positions is a characteristic effect of the early stages of oxide formation, when the oxygen atoms become chemisorbed on the $\mathrm{Fe}(110)$ surface and the $\mathrm{O}-\mathrm{FeO}(110)$ surface structure slowly evolve towards the occurrence of an oxide-like monolayer. The effect of adsorption is described in terms of the interlayer relaxation and buckling parameters, $\Delta_{i j}$ and $b_{i}$ respectively. The interlayer relaxation was computed as a function of coverage in between the first and second layers, $\Delta_{12}$, and second and third layers, $\Delta_{23}$, as shown in Figure $4 \mathrm{a}$. We found that the first-to-second interlayer relaxation transitioned from negative (O-free surface) to positive for increasing O-coverage. Thus the adsorption of oxygen induces an expansion between the first two layers, relative to bulk Fe. The interlayer relaxation parameter shifts from $\Delta_{12}=-0.37 \%$ for the O-free $\mathrm{Fe}(110)$ surface [38] to $\Delta_{12}=2.02 \%$ at $1 \mathrm{ML}$ coverage. For coverages ranging $0<\theta_{\mathrm{O}}<1 \mathrm{ML}$, there is noticeable buckling in the first layer, $b_{1}$, in the [110] direction induced by the $\mathrm{O}$ atoms bound at the $3 \mathrm{f}$ sites, as illustrated in Figure 4b. The 
oxygen atoms seem to be pushing the Fe atoms in the O-local environment on the (110) surface layer towards the bulk. At the lowest coverage, there is the greatest buckling at $0.08 \AA$. This value does not change rapidly with coverage, until we reach $1 \mathrm{ML}$ for which there is no buckling present. This decrease and collapse in the buckling is expected as at the full monolayer coverage, the 'pushing' from the oxygen atoms is uniform throughout the surface, and therefore the buckling observed at lower degrees of O-coverage is suppressed for $\theta_{\mathrm{O}}=1.0 \mathrm{ML}$. There is a slight oscillation in the interlayer relaxation parameter in between the second and third layer, $\Delta_{23}$, with respect to coverage. However, its value does not reveal a clear trend from clean to $1 \mathrm{ML}$ coverage. It is likely that the small change in between these coverages is simply a compensatory effect from the shifting in the first two layers. This statement is supported by the negligible buckling observed in the second layer, $b_{2}$, for all studied coverages. Thus it is clear that the adsorbed oxygen only strongly interacts with the first layer for an O-covered $\mathrm{Fe}(110)$ surface.

\section{Conclusions}

In this work, we have presented ab initio calculations simulating the early stages of oxidation on the (110) surface of bcc $\alpha$-iron, namely the oxygen chemisorption and the incipient formation of an $\mathrm{FeO}(111)$-like monolayer. We found the quasi-threefold $3 \mathrm{f}$ site to be most stable of all four high-symmetry adsorption sites for all O-coverages. For oxygen bound at the $3 f$ site, adsorption energies range from $\sim 2.8$ to $4.0 \mathrm{eV}$ per $\mathrm{O}$ atom for variable coverage. For low coverage, the quasi-threefold and the long-bridge (lb) sites are similar in energy, but the former becomes more favorable with increasing coverage. The short-bridge (sb) and ontop (ot) sites are highly unfavorable in terms of energy. This conclusion is re-enforced in the calculation of the vibrational frequencies, showing that the $3 f$ site is a true minimum for all coverages, whilst the $\mathrm{lb}$ constitutes a transition state. The sb and ot are rank-two saddle points. The O-adsorption induces a relaxation of the first-to-second interlayer distance with 
increasing coverage, and simultaneously a buckling effect in the first layer of the $\mathrm{Fe}(110)$ surface. The second and third layers are less sensitive to surface effects due to the O-adsorption. At full coverage the buckling in the first layer vanishes, while the relaxation parameter is highest. The analysis of the vibrational frequencies at full O-coverage yields values for the stretching and translational modes that are in good agreement with the experimental values for bulk FeO. This is supported by the increasing hybridization of the oxygen $2 p$ and iron $3 d$ orbitals with increasing oxygen coverage, and a close resemblance of the partial density of states for the O-Fe(110) system at full coverage with the one reported in the literature for bulk $\mathrm{FeO}$. These results highlight the formation of an $\mathrm{FeO}(111)$-like monolayer at full oxygen coverage, that will eventually lead to the bulk FeO surface oxide layer with increasing oxygen uptake and diffusion into the bulk.

\section{Acknowledgements}

We gratefully acknowledge the financial support of the Engineering and Physical Sciences Research Council UK (EPSRC) through the Centre for Doctoral Training in Advanced Metallic Systems. We also thank the Dalton Cumbrian Facility, partly funded by the Nuclear Decommissioning Authority, for providing funding to cover the cost of computational time. 


\section{References}

[1] Freund, H. and Pacchioni, G. (2008) Oxide ultra-thin films on metals: new materials for the design of supported metal catalysts. Chem. Soc. Rev. 37(10) 2224-2242.

[2] Ramesh, R. and Schlom, D. (2008) Whither oxide electronics? MRS Bull. 33(11) 1006-1014.

[3] Fox, K., Hoffman, E., Manjooran, N. and Pickrell, G. (2010) Advances in Materials Science for Environmental and Nuclear Technology: Ceramic Transactions John Wiley and Sons.

[4] Dorfeld, W., Hudson, J. and Zuhr, R. (1976) The interaction of an $\mathrm{O}_{2}$ molecular beam with an Fe (110) surface. Surf. Sci. 57(2) 460-474.

[5] Lawless, K. R. (1974) The oxidation of metals. Rep. Prog. Phys. 37(2) 231-316.

[6] Cabrera, N. and Mott, N. F. (1949) Theory of the oxidation of metals. Rep. Prog. Phys. 12(1) 163184.

[7] Leygraf, C. and Ekelund, S. (1973) A LEED-AES study of the oxidation of $\mathrm{Fe}(110)$ and $\mathrm{Fe}(100)$. Surf. Sci. 40(3) 609-635.

[8] Williams, A. G. and Scherer, M. M. (2004) Spectroscopic evidence for Fe (II)-Fe (III) electron transfer at the iron oxide-water interface. Environ. Sci. Technol. 38(18) 4782-4790.

[9] Seybolt, A. U. (1963) Oxidation of metals. Adv. Phys. . 12(45) 1-43.

[10] Zhou, G. (2010) Nucleation-induced kinetic hindrance to the oxide formation during the initial oxidation of metals. Phys. Rev. B. 81(19) 195440(1)-195440(7).

[11] Klueh, R. L. (2009) Ferritic/martensitic steels for advanced nuclear reactors. T. Indian I. Metals. 62(2) $81-87$.

[12] Young, D. (2008) High temperature oxidation and corrosion of metals Elsevier.

[13] Birks, N., Meier, G. H. and Petti, F. S. (2006) Introduction to the high temperature oxidation of metals Cambridge University Press.

[14] Pirug, G., Brodén, G. and Bonzel, H. P. (1980) Coadsorption of potassium and oxygen on Fe(110). Surf. Sci. 94(2) 323-338

[15] Miyano, T., Sakisaka, Y., Komeda, T. and Onchi, M. (1986) Electron energy-loss spectroscopy study of oxygen chemisorption and initial oxidation of Fe(110). Surf. Sci. 169(1) 197-215. 
[16] Hodgson, A., Wight, A. and Worthy, G. (1994) The kinetics of $\mathrm{O}_{2}$ dissociative chemisorption on Fe(110). Surf. Sci. 319(1) 119-130.

[17] Pignocco, A. J. and Pellissier, G. E. (1967) LEED studies of oxygen adsorption and oxide formation on an (011) iron surface. Surf. Sci. 7(3) 261-278.

[18] Wight, A., Condon, N. G., Leibsle, F. M., Worthy, G. and Hodgson, A. (1995) Initial stages of Fe(110) oxidation at 300 K: kinetics and structure. Surf. Sci. 331(A) 133-137.

[19] Smentkowski, V. S. and Yates, J. T. (1990) The adsorption of oxygen on Fe(110) in the temperature range of 90 to 920 K. Surf. Sci. 232(1) 113-128.

[20] Schüller, A., Busch, M., Seifert, J., Wethekam, S., Winter, H. and Gärtner, K. (2009) Superstructures of oxygen and sulphur on a $\mathrm{Fe}(110)$ surface via fast atom diffraction. Phys. Rev. B. 79(23) 235425(1)-235425(15).

[21] Weissenrieder, J., Göthelid, M., Månsson, M., von Schenck, H., Tjernberg, O. and Karlsson, U. O. (2003) Oxygen structures on Fe(110). Surf. Sci. 527(1) 163-172.

[22] Soldemo, M., Niu, Y., Zakharov, A., Lundgren, E. and Weissenrieder, J. (2015) A well-ordered surface oxide on Fe(110). Surf. Sci. 639 13-19.

[23] Busch, M., Gruyters, M. and Winter, H. (2006) FeO(111) formation by exposure of $\mathrm{Fe}(110)$ to atomic and molecular oxygen. Surf. Sci. 600(13) 2778-2784.

[24] Sakisaka, Y., Komeda, T., Miyano, T., Onchi, M., Masuda, S., Harada, Y., Yagi, K. and Kato, H. (1985) Angle-resolved photoemission of the c(2x2) and c(3x1) oxygen overlayers on $\mathrm{Fe}(110)$. Surf. Sci. 164(1) 220-234.

[25] Kim, H.-J. and Vescovo, E. (1998) Spin-resolved photoemission investigation of the $c(2 \times 2)$ and $c(3 \times 1)$ oxygen overlayers on the Fe(110) surface. Phys. Rev. B. 58(20) 14047-14050.

[26] Poling, G. W. (1969) Infrared Reflection Studies of the Oxidation of Copper and Iron. $J$.

Electrochem. Soc. 116(7) 958-963.

[27] Erley, W. and Ibach, H. (1981) Vibrational excitations and structure of oxygen on Fe(110). Solid State Commun. 37(12) 937-942.

[28] de Faria, D. L. A., Silva, S. V. and de Oliveira, M. T. (1997) Raman Microspectroscopy of Some Iron Oxides and Oxyhydroxides. J. Raman Spectrosc. 28 873-878. 
[29] Eder, M., Terakura, K. and Hafner, J. (2001) Initial stages of oxidation of (100) and (110) surfaces of iron caused by water. Phys. Rev. B: Condens. Matter. 64(11) 1-7.

[30] Błoński, P., Kiejna, A. and Hafner, J. (2005) Theoretical study of oxygen adsorption at the Fe(110) and (100) surfaces. Surf. Sci. 590(1) 88-100.

[31] Błoński, P., Kiejna, A. and Hafner, J. (2007) Oxygen adsorption on the clean and O precovered Fe(110) and (100) surfaces. J. Phys.: Condens. Matter. 19(9) 1-8.

[32] Błoński, P., Kiejna, A. and Hafner, J. (2008) Dissociative adsorption of $\mathrm{O}_{2}$ molecules on Oprecovered Fe(110) and Fe (100): Density-functional calculations. Phys. Rev. B: Condens. Matter. 77(15) $1-8$.

[33] Tan, X., Zhou, J. and Peng, Y. (2012) First-principles study of oxygen adsorption on Fe(110) surface. Appl. Surf. Sci. 258(22) 8484-8491.

[34] Ossowski, T. and Kiejna, A. (2015) Oxygen adsorption on Fe(110) surface revisited. Surf. Sci. $63735-41$.

[35] Freindl, K., Ossowski, T., Zając, M., Spiridis, N., Wilgocka-Ślęzak, D., Madej, E., Giela, T., Kiejna, A. and Korecki, J. (2016) Oxygen Adsorption on the Fe (110) Surface: The Old System-New Structures. J. Phys. Chem. C. 120(7) 3807-3813.

[36] Clark, S. J., Matthew, I., Segall, D., Pickard, C. J., Hasnip, P. J., Probert, M. I. J., Refson, K. and Payne, M. C. (2005) First principles methods using CASTEP. Z. Kristallogr. 220 567-570.

[37] Perdew, J. P., Burke, K. and Ernzerhof, M. (1996) Generalized Gradient Approximation Made Simple. Phys. Rev. Lett. 77(18) 3865-3868.

[38] Chohan, U. K., Jimenez-Melero, E. and Koehler, S. P. K. (2016) Surface atomic relaxation and magnetism on hydrogen-adsorbed Fe (110) surfaces from first principles. Appl. Surf. Sci. 387 385392.

[39] Pack, J. D. and Monkhorst, H. J. (1976) Special points for Brillouin-zone integrations. Phys. Rev. B: Condens. Matter. 13(12) 5188-5192.

[40] De Vita, A. (1992) The energetics of defects and impurities in metals and ionic materials from first principles. University of Keele. 
[41] Pfrommer, B. G., Côté, M., Louie, S. G. and Cohen, M. L. (1997) Relaxation of crystals with the quasi-Newton method. J. Comput. Phys. 131(1) 233-240.

[42] Morris, A., Nicholls, R., Pickard, C. and Yates, J. (2014) OptaDOS: A tool for obtaining density of states, core-level and optical spectra from electronic structure codes. Comput. Phys. Commun. 185(5) 1477-1485.

[43] Yates, J., Wang, X., Vanderbilt, D. and Souza, I. (2007) Spectral and Fermi surface properties from Wannier interpolation. Phys. Rev. B. 75(19) 195121.

[44] Kratzer, P., Hammer, B. and No, J. (1996) A theoretical study of $\mathrm{CH}_{4}$ dissociation on pure and gold alloyed Ni (111) surfaces. J. Chem. Phys. 105(13) 5595-5604.

[45] Stull, D. R. and Prophet, H. (1971) JANAF thermochemical tables.

[46] Hammer, B. and Nørskov, J. (1995) Electronic factors determining the reactivity of metal surfaces. Surf. Sci. 343(3) 211-220.

[47] Terakura, K., T, O., Williams, A. and Kübler, J. (1984) Band theory of insulating transition-metal monoxides: Band-structure calculations. Phys. Rev. B. 30(8) 4734-4747.

[48] Ye, L., Asahi, R., Peng, L. and Freeman, A. (2012) Model GW study of the late transition metal monoxides. J. Chem. Phys. 137(15 ) 186401(1)-186401(5).

[49] Rödl, C., Fuchs, F., Furthmüller, J. and Bechstedt, F. (2009) Quasiparticle band structures of the antiferromagnetic transition-metal oxides $\mathrm{MnO}, \mathrm{FeO}, \mathrm{CoO}$, and NiO. Phys. Rev. B. 79(23) 235114(1)$235114(8)$ 


\section{Tables}

Table 1. Wavenumbers of the $\mathrm{Fe}-\mathrm{O}$ vibrations at the four studied adsorption sites on the $\mathrm{Fe}(110)$ surface, for representative cell geometries, with only one $\mathrm{O}$ atom adsorbed per cell. $\tilde{v}_{\mathrm{t} 1}$ and $\tilde{v}_{\mathrm{t} 2}$ are the frustrated translational modes, oscillating in the [1 $\left.\overline{1} 0\right]$ and [001] directions, respectively. $\tilde{v}_{s}$ is the stretching frequency, oscillating in the [110] direction.

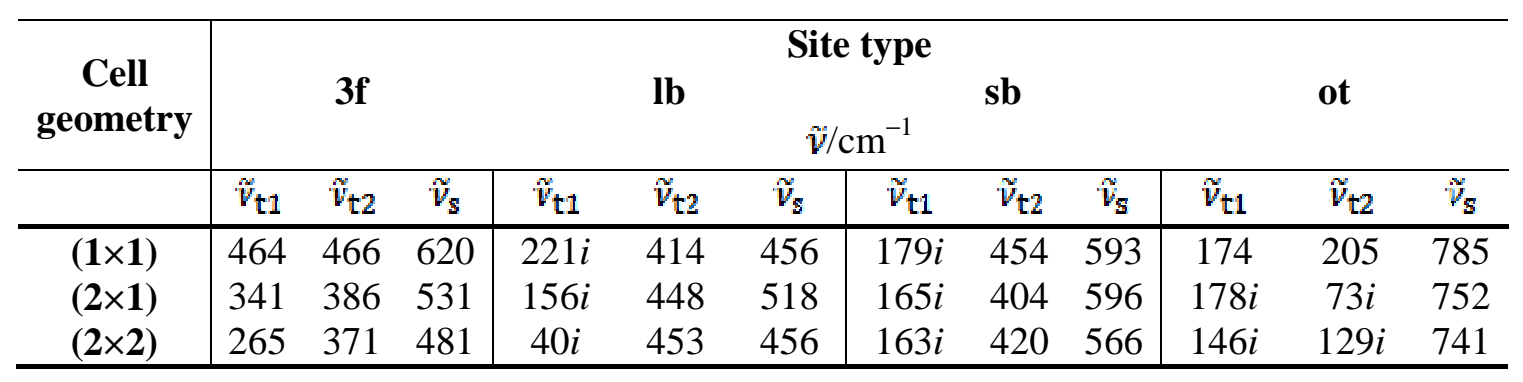

Table 2. Wavenumbers of the $\mathrm{Fe}-\mathrm{O}$ vibrations for oxygen located at the $3 \mathrm{f}$ adsorption site, for all studied combinations of cell geometries and coverages $\left(\theta_{\mathrm{O}}\right)$.

\begin{tabular}{c|c|ccc}
\hline Cell geometry & $\theta_{\mathrm{O}} / \mathrm{ML}$ & \multicolumn{3}{|c}{$\tilde{v}^{\prime} / \mathrm{cm}^{-1}$} \\
$\tilde{v}_{\mathrm{t} 2}$ & $\tilde{v}_{\mathbf{s}}$ \\
\hline $\mathbf{( 2 \times 2 )}$ & 0.25 & 265 & 371 & 481 \\
$\mathbf{( 3 \times 1 )}$ & 0.33 & 351 & 380 & 521 \\
$(\mathbf{2} \times \mathbf{1})$ & 0.50 & 341 & 386 & 531 \\
$(\mathbf{2} \times \mathbf{2})-\mathbf{2 O}$ & 0.50 & $313 / 339$ & $393 / 426$ & $501 / 530$ \\
$(\mathbf{3} \times \mathbf{1})-\mathbf{2 O}$ & 0.67 & $331 / 408$ & $410 / 419$ & $521 / 558$ \\
$(\mathbf{1} \times \mathbf{1})$ & 1.00 & 464 & 466 & 620 \\
\hline
\end{tabular}




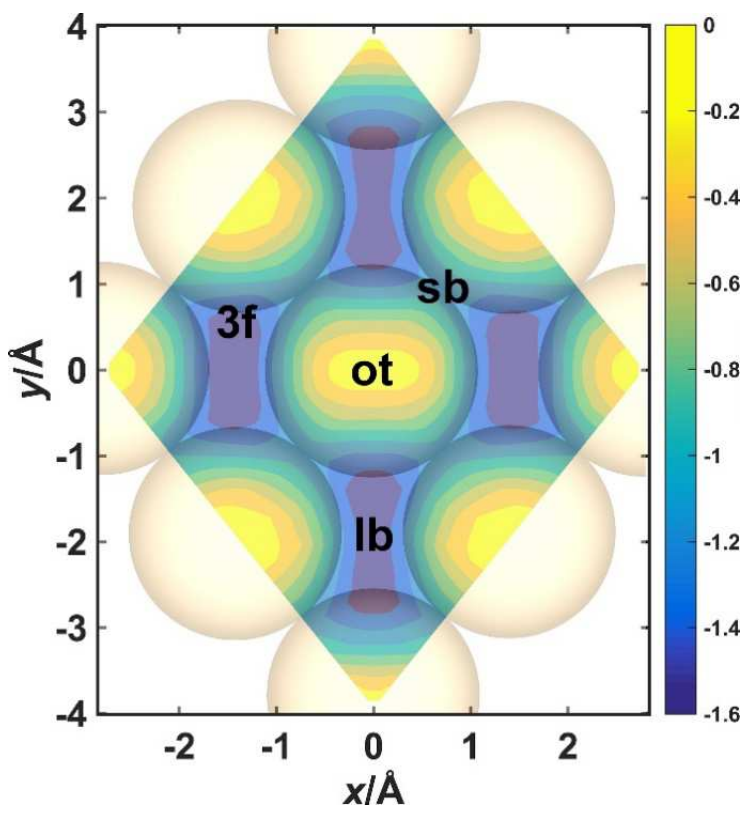

Figure 1. The potential energy surface for oxygen adsorbed on the $\mathrm{Fe}(110)$ surface, at and in between the various high symmetry adsorption sites. The adsorption sites for $\mathrm{O}$ atoms on the $\mathrm{Fe}(110)$ plane are depicted as follows: the quasi-threefold (3f), long-bridge (lb), short-bridge $(\mathrm{sb})$, and on-top (ot) sites. The $(2 \times 2)$ geometry was selected to generate the potential energy surface. 


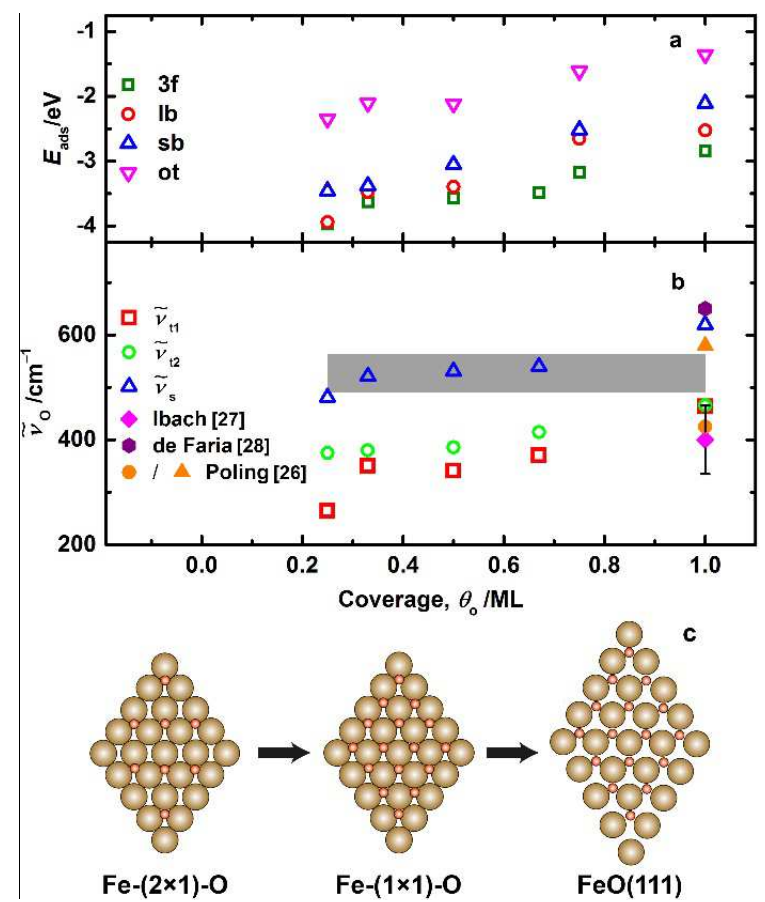

Figure 2. (a) Calculated adsorption energies at four high symmetry adsorption sites, and (b) vibrational frequencies at the $3 \mathrm{f}$ site for $\mathrm{O}$ adsorbed on $\mathrm{Fe}(110)$ at various coverages. Closed symbols depict the experimental values from previous experimental work. The grey band depicts the range of vibrational frequencies for varying coverages of adsorbed oxygen as measured by HREEL in Ref. [27]. (c) The surface arrangements for half and full monolayer oxygen coverage, and the arrangement of atoms to form the $\mathrm{FeO}(111)$ monolayer. 

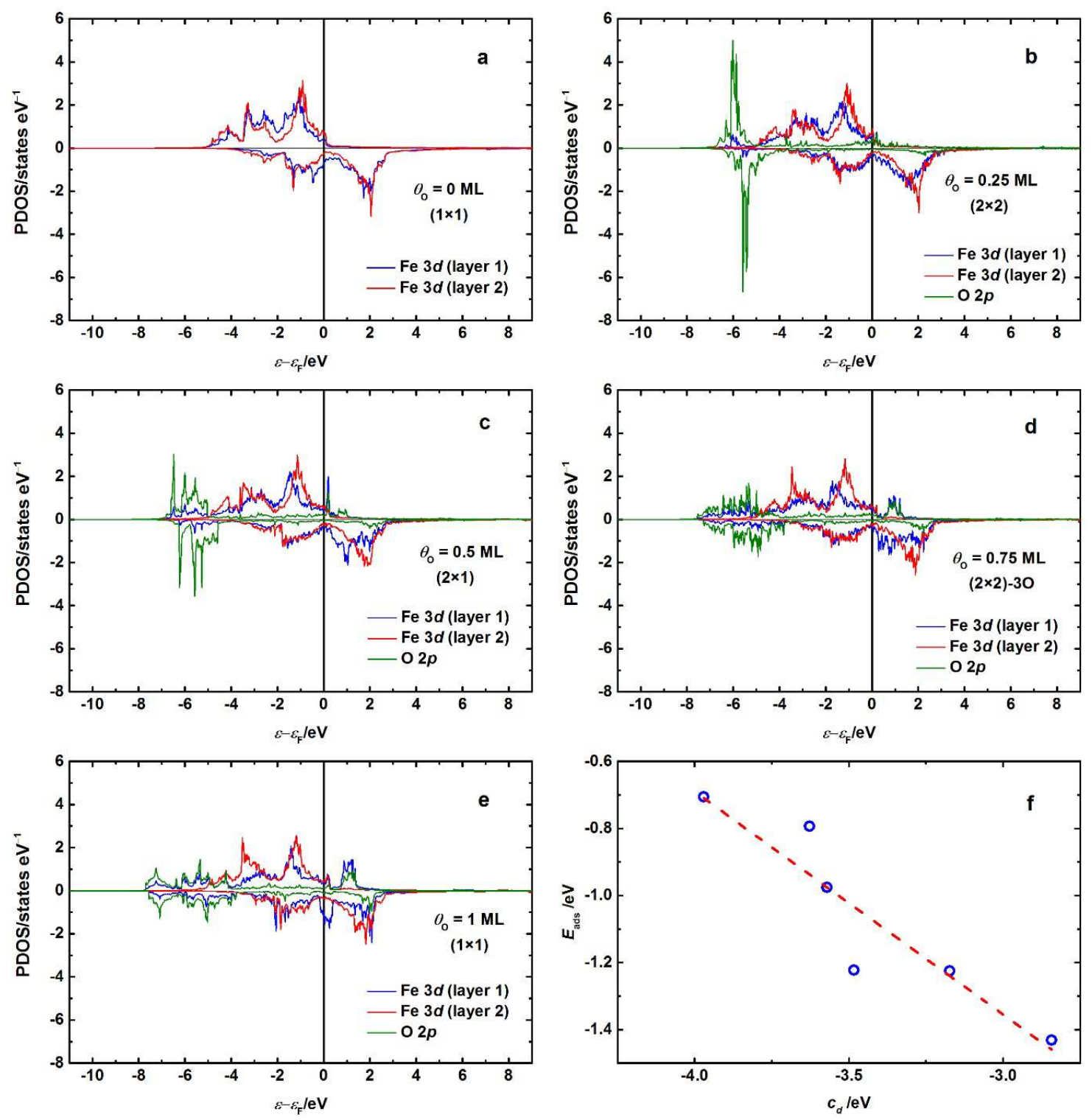

Figure 3. (a)-(e) Spin-polarized partial density of states (PDOS) of oxygen adsorption on $\mathrm{Fe}(110)$ at the $i$ th layer $(i=1,2)$ for increasing coverage; from 0-1 ML (in 0.25 ML intervals). The vertical line denotes the Fermi energy, $E_{\mathrm{F}}$. (f) Adsorption energy, $E_{\mathrm{ads}}$, as a function of the $d$-band center, $c_{d}$, the latter calculated from the PDOS. The dashed line is a guide to the eye. 


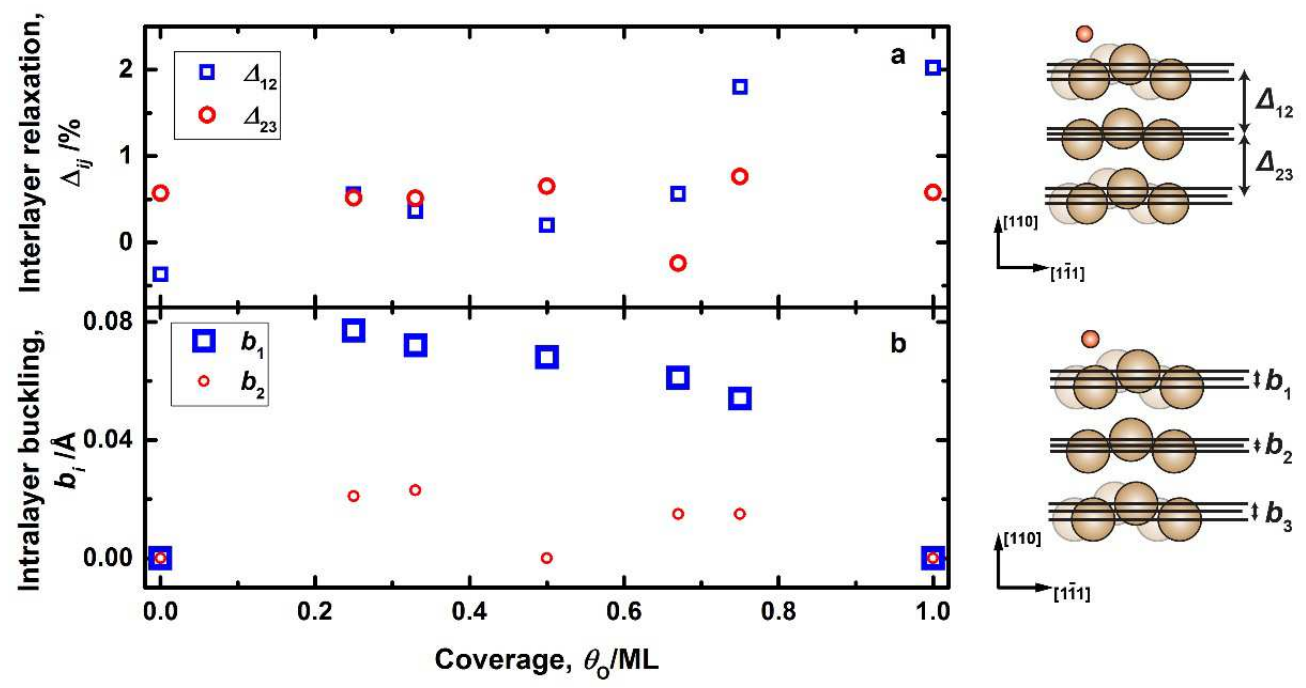

Figure 4. (a) The interlayer relaxation $\Delta_{i j}$ between layers $i$ and $j$ of the $\mathrm{Fe}(110)$ surface, together with (b) intralayer buckling $b_{i}$, as a function of coverage. 